\title{
Immune status in sarcoidosis: one size does not fit all
}

\author{
Ilias C. Papanikolaou ${ }^{1}$, Hidenobu Shigemitsu ${ }^{2}$, Andreas Afthinos ${ }^{1}$ \\ ${ }^{1}$ Pulmonary Department, General Hospital of Corfu, Greece; ${ }^{2}$ Department of Intensive Care Medicine, Tokyo Medical \\ and Dental University, Tokyo, Japan
}

\begin{abstract}
B-cell immunity and immunoglobulins are less commonly affected in sarcoidosis. We aimed to evaluate immune status in sarcoidosis patients. Retrospective chart review of sarcoidosis patients attending a out-patient clinic over 3 months period. Immunoglobulins levels were recorded (A, M, G, E) along with clinical and serological data. They were divided in Group A (normal IgG), Group B (increased IgG), Group C (decreased IgG) and Group D (decreased IgG and IgM and/or IgA). Of 50 subjects, $68 \%$ were females and $62 \%$ of Caucasian origin. $22(44 \%)$ had normal IgG levels, 16 (32\%) had increased IgG levels, $10(20 \%)$ had hypogammaglobulinemia and 2 (4\%) had combined hypogammaglobulinemia, diagnosed with combined sarcoidosis and common variable immunodeficiency. Decreased IgA values was found in Groups C and D. IgE was high in Group B. Globulin was increased in Group B and decreased in Groups C and D.
\end{abstract}

Correspondence: Ilias C. Papanikolaou, Department of Pulmonary Medicine, Corfu General Hospital, 1 Kontokaliou street, Corfu 49100, Greece.

Tel. +30.2661360695 .

Fax: +30.2661033088 .

E-mail: icpapanikolaou@hotmail.com

Key words: Sarcoidosis; immunity; interstitial lung disease.

Contributions: IP and HS collected the data. IP, HS and AA all analyzed the data and drafted the manuscript. All the authors have read and approved the final version of the manuscript and agreed to be accountable for all aspects of the work.

Conflict of interest: The authors declare no conflict of interest.

Funding: None.

Ethics approval: The study was approved by the ethics committee of the University of Southern California University Hospital.

Received for publication: 14 April 2020.

Accepted for publication: 25 May 2020

${ }^{\circ}$ Copyright: the Author(s), 2020

Licensee PAGEPress, Italy

Monaldi Archives for Chest Disease 2020; 90:1306

doi: 10.4081/monaldi.2020.1306

This article is distributed under the terms of the Creative Commons Attribution Noncommercial License (by-nc 4.0) which permits any noncommercial use, distribution, and reproduction in any medium, provided the original author(s) and source are credited.
Decreased neutrophils were found in Group D (all statistically significant). Correlation analysis showed significant association of angiotensin converting enzyme with $\operatorname{IgA}$ and $\operatorname{IgM}$, inverse correlation of $\operatorname{IgG}$ with white blood cells and neutrophils, of IgA with globulin and inverse with albumin and of calcium with albumin. Most sarcoidosis patients have normal or increased immunoglobulin levels, that correlate with serum biomarkers of disease activity. Hypogammaglobulinemia may reflect treatment side effects and accompanied by blood leukocytosis. Combined severe immunodeficiency occurs in sarcoidosis.

\section{Introduction}

Sarcoidosis is a multi-system disease of unknown origin characterized by granulomatous inflammation. Sarcoid granulomas are non-caseating and consist of macrophages, epithelioid cells, multi-nucleated giant cells and T-lymphocytes. It is believed that certain antigen(s) through antigen presenting cells activate T-lymphocytes to an oligoclonal mainly CD4 Thelper-1 expansion (but also Th-2 and Th-17) that via IFN- $\gamma$ and other cytokines lead to granuloma formation [1].

B-cells are less activated, and immunoglobulins (Ig's) in sarcoidosis are commonly found normal or, less commonly, elevated [2]. Decrease of immunoglobulins is thus not a characteristic of sarcoidosis; there are reports however of sarcoidosis co-incidence with common variable immunodeficiency (CVID) [3,4]. We sought to investigate immune status in our sarcoidosis patients, in particular the incidence of hypogammaglobulinemia. We further examined Ig's status in relation to white blood cells and various serum proteins.

\section{Materials and Methods}

This was a retrospective chart review of sarcoidosis patients attending outpatient sarcoidosis clinic at University of Southern California University Hospital from September 2013 until December 2013. The study was approved by the ethics committee of USC-UH. Charts were reviewed by the investigators and they recorded Igs ( $\mathrm{G}, \mathrm{A}, \mathrm{M}, \mathrm{E})$, angiotensin converting enzyme (ACE), globulins, albumin, white blood cell counts and differential, calcium and treatment regimens. Inclusion criteria was a diagnosis of sarcoidosis according to established guidelines (compatible with sarcoidosis clinical presentation, demonstration of non-caseating granuloma on tissue biopsy and exclusion of other diagnoses) [5]. Exclusion criteria were a diagnosis of primary hypogammaglobulinemia, protein-losing enteropathies and causes of secondary hypogammaglobulinemia (thymoma, hematological malignan- 
cies, systemic illnesses). Among 50 sarcoidosis subjects recruited, according to Scadding's radiology staging system, 3 (6\%) were stage $0,10(20 \%)$ were stage I, $18(36 \%)$ were stage II, $13(26 \%)$ were stage III and $6(12 \%)$ were stage IV.

\section{Definition of immunoglobulins}

Quantification of IgG, IgA, IgM and IgE was accomplished with nephelometry. IgG normal range was $768-1632 \mathrm{mg} / \mathrm{dL}$, IgA $68-378 \mathrm{mg} / \mathrm{dL}$, IgM $60-263 \mathrm{mg} / \mathrm{dL}$ and IgE $<100 \mathrm{mg} / \mathrm{dL}$. Based on Ig's values, accounting primarily for $\mathrm{IgG}$, we sub-classified sarcoidosis subjects in 4 groups: A, with normal IgG levels, B, with elevated IgG levels (hypergammaglobulinemia), $\mathrm{C}$, with decreased IgG levels (hypogammaglobulinemia), and D with decreased IgG levels + decrease in IgA and/or IgM (possible CVID).

Diagnosis of CVID requires low serum IgG total concentration along with low serum IgA and/or IgM, poor response to immunization and exclusion of other immunodeficiency diseases [6].

\section{Statistical analysis}

Parameters between these sub-groups were evaluated with non-parametric two-tailed ANOVA test (Kruskall-Wallis). GraphPadPrism 8.3.1 software was used.

\section{Results}

Among 50 study subjects included in the study, 34 (68\%) were females. Race distribution follows: 31 patients were Caucasians,
14 African-Americans, 4 Hispanics and 1 Asian. 22 patients were classified as Group A, 16 at Group B, 10 at Group C, 2 at Group D (Table 1$) .30 / 50$ patients $(60 \%)$ were on treatment mainly with corticosteroids. Among them, 15 were from Group A, 9 from Group B and 6 from Group C.

Decreased $\operatorname{IgA}$ was found in Groups $\mathrm{C}$ and D. $\operatorname{IgE}$ was high in Group B. As expected, globulin was increased in Group B and decreased in Groups C and D. Relatively decreased neutrophils were found in Group D and increased in C (all statistically significant).

Correlation analysis in the total study subjects showed significant association of ACE with IgA and IgM, inverse correlation of IgG with white blood cells and neutrophils, correlation of IgA with globulin and inversely with albumin and, correlation of calcium with albumin.

\section{Discussion}

As assessed by immunoglobulins, among our sarcoidosis cases, $44 \%$ had normal immune status, 32\% had hypergammaglobulinemia and $24 \%$ had hypogammaglobulinemia (Groups C and D combined). In this last group, $4 \%$ of total study group possibly fulfill criteria for a diagnosis of CVID.

Hypergammaglobulinemia is reported to occur in $30-80 \%$ of sarcoidosis patients, a finding of our study as well. Interestingly, these immune-stimulated patients exhibited increased other immunoglobulins (specifically $\operatorname{IgE}$ ) and also increased serum ACE correlating with immunoglobulins $\mathrm{A}$ and $\mathrm{M}$. This is a novel finding. ACE is a glycoprotein produced by different tissues and its serum concentrations may reflect monocyte-macrophage system activity [7]. ACE activity is elevated in lungs of sarcoidosis patients, secondary to the macrophage alveolitis and enhanced expression of epithelial cells [8]. Since there is a limited B-cell expansion in sarcoidosis, it is possible that disease activity corre-

Table 1. Demographic, immunoglobulins and blood characteristics of study subjects.

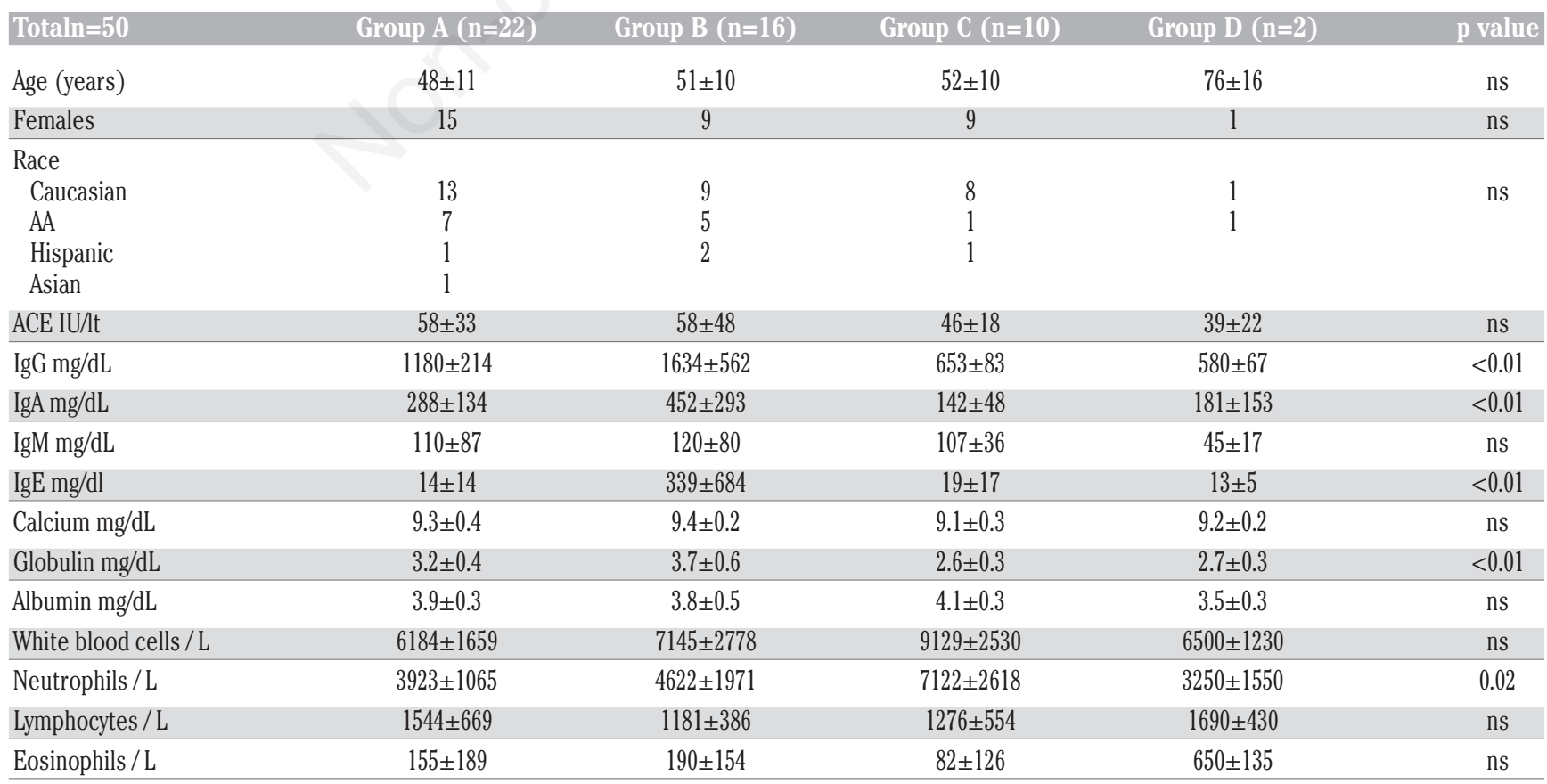

ns, not significant. 
lates with hyper-immunity translated ultimately in increased immunoglobulin levels.

On the other hand, hypogammaglobulinemia raises the suspicion for CVID and in general has to be investigated. Cases of sarcoidosis-CVID coexistence are reported in the literature. CVID may also be misdiagnosed as sarcoidosis, particularly when pulmonary disease manifests in the form of granulomatous-lymphocytic interstitial lung disease (GL-ILD) [9]. We chose to separate Groups C and D. Cases of hypogammaglobulinemia without any other immunoglobulin deficiency (Group C), may be considered, to our view, as secondary to corticosteroids hypogammaglobulinemia [10]. In our study, all subjects of Group C were on corticosteroids, either at the time of study or had stopped them the last 6 months. In particular, such secondary effect of corticosteroids is reported to affect mainly $\operatorname{IgA}$ and not $\operatorname{IgM}$, exactly the same with our Group C cases.

When hypogammaglobulinemia is accompanied by one more immunoglobulin deficiency, then a diagnosis of CVID is very likely. We found two such cases (Group D). Interestingly none of them was on corticosteroids. We consider sarcoidosis with late CVID diagnosis in these two cases.

A novel finding, to our knowledge, is the inverse association of IgG with white blood cells and neutrophils. Increased neutrophils in Group $\mathrm{C}$ may be related to treatment with corticosteroids. Moreover, neutrophils were decreased in Group D of sarcoidosis and CVID, though in a very limited number of patients $(n=2)$. The former has been reported in patients with hematologic malignancies. Functional studies may clarify better this association of globulins and hematologic phenotypes.

The majority of our study subjects had pulmonary sarcoidosis (stages II, III and IV). Cluster studies have previously shown that pulmonary involvement is associated with chronic disease, older age, increased likelihood of immunosuppressive treatment and systemic features like hepato-splenic involvement [11,12]. To what extent specific phenotypes (especially the latter) affect immunity needs further investigation.

Immunoglobulins studies in sarcoidosis are lacking. We presented here an analysis of 50 patients, adding hematological profile data. More sophisticated studies using peripheral blood monocytes and IgG subclasses could further elucidate this heterogeneity in sarcoidosis patients.

\section{Conclusions}

Most of sarcoidosis patients have normal or increased immunoglobulin levels, that may correlate with other serum biomarkers of disease activity. Hypogammaglobulinemia is frequent, may reflect treatment side effects and accompanied by blood leukocytosis. Combined severe immunodeficiency occurs in sarcoidosis or even masquerades sarcoidosis. Immunological status varies among sarcoidosis patients and may have significant treatment implications.

\section{References}

1. Forrester JM, Wang Y, Ricalton N, et al. TCR expression of activated $\mathrm{T}$ cell clones in the lungs of patients with pulmonary sarcoidosis. J Immunol 1994; 153:4291.

2. Belhomme N, Jouneau S, Bouzillé G, et al. Role of serum immunoglobulins for predicting sarcoidosis outcome: A cohort study. PLoS One 2018; 13:e0193122.

3. Fasano MB, Sullivan KE, Sarpong SB, et al. Sarcoidosis and common variable immunodeficiency. Report of 8 cases and review of the literature. Medicine (Baltimore) 1996;75:251-61.

4. Mechanic LJ, Dikman S, Cunningham-Rundles C. Granulomatous disease in common variable immunodeficiency. Ann Intern Med 1997;127: 613-7.

5. Hunninghake GW, Costabel U, Ando M, et al. Sharma OP: ATS/ERS/WASOG statement on sarcoidosis. Sarcoidosis Vasc Diffuse Lung Dis 1999;16:149-73.

6. Chapel H, Lucas M, Lee M, et al. Common variable immunodeficiency disorders: division into distinct clinical phenotypes. Blood 2008;112:277.

7. Betrains A, Vermeersch P, Vanderschueren S. Appropriateness of ordering serum angiotensin-converting enzyme during renin-angiotensin-aldosterone system inhibitor therapy. Eur J Intern Med 2019;59:e18-9.

8. d'Alessandro M, Bergantini L, Perrone A, et al. Serial investigation of Angiotensin-Converting Enzyme in sarcoidosis patients treated with Angiotensin-Converting Enzyme Inhibitor. Eur J Intern Med 2020. doi: 10.1016/j.ejim.2020. 04.006 .

9. Bates CA, Ellison MC, Lynch DA, et al. Granulomatous-lymphocytic lung disease shortens survival in common variable immunodeficiency. J Allergy Clin Immunol 2004;114:415.

10. Fedor ME, Rubinstein A. Effects of long-term low-dose corticosteroid therapy on humoral immunity. Ann Allergy Asthma Immunol 2006;97:113.

11. Rubio-Rivas M, Corbella X. Clinical phenotypes and prediction of chronicity in sarcoidosis using cluster analysis in a prospective cohort of 694 patients. Eur J Intern Med 2020. doi:10.1016/j.ejim.2020.04.024.

12. Pérez-Alvarez R, Brito-Zerón P, Kostov B, et al. Systemic phenotype of sarcoidosis associated with radiological stages. Analysis of 1230 patients. Eur J Intern Med 2019;69:77-85. 